\section{Converting Right-Left Stereo Pairs Into Colored Pairs For Electronic Presentation}

Mark W. Tengowski

Pfizer Inc.

mark_w_tengowski@groton.pfizer.com

Since Microsoft PowerPoint has become the dominant scientific meeting presentation format, my collection of right-left stereo slide pairs has basically been rendered obsolete. Yet, contained within these striking images are valuable scientific data. This communication describes an easy method whereby analog slide data (i.e. scanning electron micrograph RL pairs, Figs. 1 \& 2) are transformed into an electronic format such that the need for projector polarizers is replaced by inexpensive red-green or red-blue 3-D glasses.

\section{Conversion of $2 \times 2$ slides into digital files}

This is easily accomplished with today's flatbed scanners (i.e. Agfa DuoScan series) or slide scanners (i.e. Nikon Super Coolscan 8000) that accommodate $35 \mathrm{~mm}$ slides. Just like orientation into the slide carousel was critical for on-screen display, scanning slides into a digital file format is critical. Use a desired scanning resolution (i.e. 300 dpi) pre-loaded with the scanner software, selecting the image from the preview scan. When scanning and naming slides, be cognizant of the right and left images, as well as the orientation needed for correct reconstruction. Rotating a scanned image during processing is a simple, but critical task, to assure final stereopsis display. Save your files in TIFF or JPEG format.

\section{Creating colored stereo pairs in Adobe Photoshop}

We will take advantage of PhotoShop's ability to separate image plates not only into layers, but also into specific color channels.

1. Open both original right and left image files. If needed, rotate each canvas to assure proper orientation and adjust gray levels. (For me, this required a $90^{\circ}$ clockwise rotation of each original image.)

2. Create a new image document with the same pixel dimensions. The mode must be RGB color for this new file. The Layers option will now show the background.

3. Copy the Left image (select all + copy) and paste into the new Layer 1.

4. Change the name of the layer, using the layer properties, to indicate Left.

5. Create a new layer (Layer|New/Layer) and rename to Right.

6. Bring the scanned right image to the active window, select all, and copy the Right image.

7. Paste this image in the new Right layer in the RGB color working file. At this time, your working file should contain a right and left layer corresponding to the scanned files, but only one image at a time is visible, depending on layer selected.

8. In this working file, select the left layer and change to the Channels tab. We will now 'colorize' the gray-scale image by suppressing two of the channels.

9. To accomplish this, select Image|Adjust|Levels to bring up a Levels dialog box. We want the left image to be red, so we change the Channel in this dialog box to Blue and reduce the output level from 255 to zero; repeat for the Green channel. You should now see this layer as a red-scale image.

10. Close the Levels dialog box by clicking $O K$.

11. Switch back to the Layers tab and select the Right layer.

12. Again, activate the Channels tab and adjust the color contribution to suppress red and blue, thereby creating a green right image.

13. Activate the Layers tab, and with the Right layer selected, reduce the opacity from $100 \%$ to $60 \%$ (provided for guidance, adjust if needed). This permits the red layer previously unseen to now be partly visible.

14. As with regular stereo pair alignment, you may need to use the Move tool to adjust the layers for correct stereo overlay.

15. You can use the Crop tool to remove the slight overhangs for scanned file mismatch.

16. Additionally, layers with arrows or micron bars can now be added.

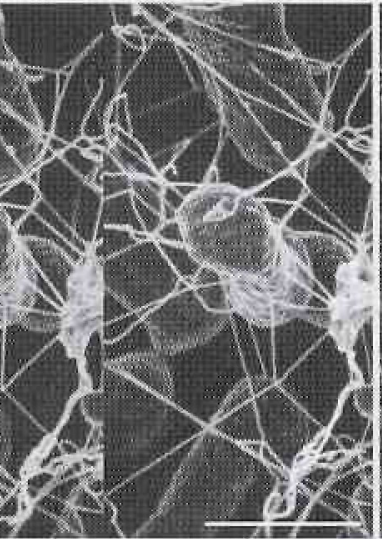

Fig. 1. E. coli in culture, bar $=860$

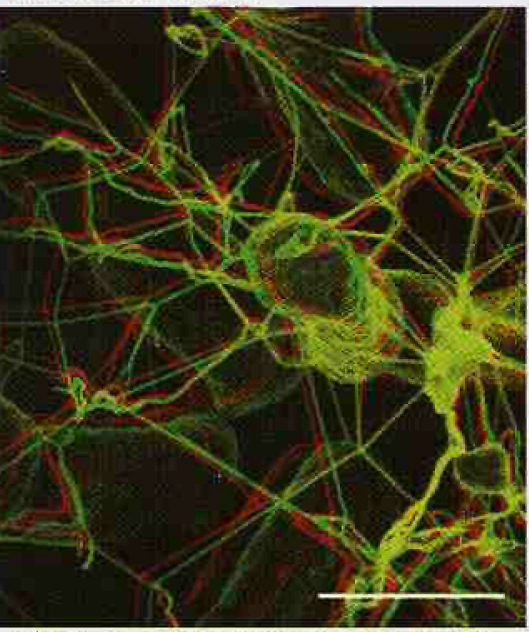

Fig. 3. E. coli in culture reconstructed as a Red-Green pair, bar $=860 \mathrm{~mm}$.

"Figure images are shown to illustrate the method and are not intended to be viewed in 3-D. Full size

Fig. 4. L. pictus recontruction as a Red-Blue pair, bar $=3$ micron.
Fig. 2. Fractured surface of a L. pictus egg, bar $=3 \mu \mathrm{m}, 10 \mathrm{kX}, 1.5 \mathrm{kV}$.*
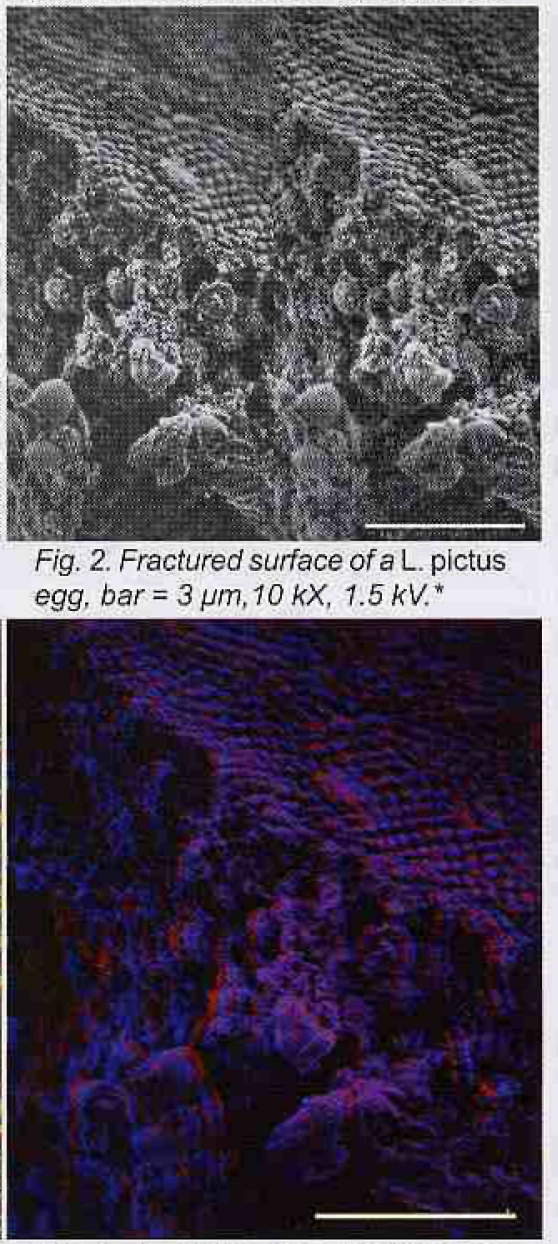$$
\text { ize }
$$

and 2, sutable for stereo viewing, are avaliable from the author or the editor.

If you plan to alter the file at a later time, it is advised that you store the image in the PhotoShop format (".psd). However, you still have the original TIFFs and can quickly reproduce the RG pair with this method. Since the output is most likely a digital projector with much lower resolution than your original scans, for the sake of file size, utilize the JPEG compression option (mid-level compression reduces file to $350 \mathrm{kB}$ ) or reduce the Document Size resolution. If you choose the JPEG option, you will need to flatten the image (Layer|Flatten Image) before the JPEG option is avallable for file saving (File/Save As). This RG procedure (Fig. 3 ) can be altered for redblue pairs (Fig. 4) by changing the right layer channels to suppress red and green. My lab has both $R G$ and $R B$ viewing glasses, so this option adds to the flexibility of the procedure. The reconstructed image file can now be inserted into your PowerPoint presentation, just as you would any other photomicrograph or graphic. N.B.: Microsoft PowerPoint is a trademark of the Microsoft Corporation; Adobe and PhotoShop are trademarks of the Adobe 